\title{
La publicité électorale de la campagne présidentielle américaine en 1992
}

Rhétorique politique et symbolique culturelle

\section{Donna Andréolle}

\section{OpenEdition}

\section{Journals}

Édition électronique

URL : http://journals.openedition.org/asp/4066

DOI : 10.4000/asp.4066

ISSN : 2108-6354

Éditeur

Groupe d'étude et de recherche en anglais de spécialité

Édition imprimée

Date de publication : 1 décembre 1994

Pagination : fr

ISSN : 1246-8185

Référence électronique

Donna Andréolle, «La publicité électorale de la campagne présidentielle américaine en 1992 », ASp [En ligne], 5-6 | 1994, mis en ligne le 09 décembre 2013, consulté le 03 mai 2019. URL : http:// journals.openedition.org/asp/4066; DOI : 10.4000/asp.4066

Ce document a été généré automatiquement le 3 mai 2019.

Tous droits réservés 


\title{
La publicité électorale de la campagne présidentielle américaine en 1992
}

Rhétorique politique et symbolique culturelle

\author{
Donna Andréolle
}

\section{Introduction au compte rendu d'une expérience de recherche}

1 L'avènement de la télévision aux États-Unis après la Deuxième Guerre mondiale a bouleversé la vie politique en général et les campagnes électorales en particulier. Dès 1952, Rosser Reeves, publiciste de renom et directeur de la campagne présidentielle de Dwight Eisenhower, mit à l'épreuve sa thèse selon laquelle « l'électeur dans l'isoloir hésite comme dans une pharmacie lorsqu'il s'agit de choisir entre deux pâtes dentifrices » (Ramonet 1989: 24). Il estimait qu'une bonne publicité électorale doit être centrée sur une "proposition unique de vente $»^{1}$, car la façon la plus efficace de vendre, selon lui, était de répéter sans cesse un seul et unique message. L'électeur choisirait donc «la marque (c'est-à-dire le candidat) dont on lui a le plus parlé » (Ramonet 1989 : 25). Ce fut la naissance du candidat-produit.

2 La publicité négative fit son apparition en 1964, pendant la campagne présidentielle qui opposait Lyndon Johnson à Barry Goldwater. La publicité en question (appelée plus tard « The Daisy Girl ad») attaquait la politique pro-nucléaire des Républicains. La stratégie était simple et efficace : dramatisation (compte à rebours, voix « d'outre-tombe » du narrateur) et visualisation (image d'une petite fille anéantie par une explosion nucléaire). S'ajoutait à cette nouvelle forme de message son mode d'emploi : saturation (le principe de répétition de Reeves) des ondes aux heures de grande écoute, puis retrait du spot en réponse aux protestations de l'opposition (Donovan \& Scherer 1992 : 228). 
3 L'objectif du présent article sera d'analyser, à travers quatre exemples de publicité électorale de la campagne présidentielle de 1992, quelques éléments de rhétorique politique conçue spécialement pour le support télévisuel ainsi que l'importance symbolique des images et de l'accompagnement musical dans la transmission du message électoral des deux principaux candidats, George Bush et Bill Clinton.

\section{Le candidat-produit : étude thématique de deux publicités positives}

D'abord, il convient de préciser que le terme "publicité positive» dénote un spot promotionnel de courte durée (30 à 60 secondes) comprenant les éléments d'une publicité classique, à savoir : l'identification du produit, la présentation de ses caractéristiques, l'image du produit et sa mise en scène ainsi que l'utilisation d'un slogan.

5 Ces publicités vantent les mérites du candidat ou visent à promouvoir un programme particulier, un programme économique par exemple. Quelle que soit la forme de la publicité, le candidat sera traité comme un bien de consommation.

\section{George Bush, tradition et continuité ${ }^{2}$}

Dans ce premier exemple, on notera d'abord que le narrateur est George Bush lui-même, qui nous présente son programme économique appelé Agenda for American Renewal. En tant que produit il est mis en scène deux fois : on le voit, à l'ouverture, dans son bureau de la Maison-Blanche (the Oval Office) et à la fin entouré de sa famille, en train de se promener dans un cadre verdoyant. Ces deux scènes permettent de créer une association entre le candidat et son image de marque : avant tout il est l'homme d'État expérimenté, symbole de l'autorité présidentielle; en deuxième lieu, il est humain, un homme de famille. Entre ces deux images du candidat-produit nous avons une succession de scènes hollywoodiennes idylliques - le monde utopique auquel nous pouvons appartenir si nous votons pour George Bush. L'accompagnement musical ${ }^{3}$ contribue au sentiment de bonheur et de bien-être évoqué par ces scènes du rêve américain tel qu'il est conçu par le Parti républicain.

7 Puisque Bush est le candidat sortant et qu'il a déjà fait huit ans en tant que Vice-Président dans l'administration Reagan, il doit opter pour les thèmes de la tradition (le patriotisme, l'autorité institutionnelle, la cellule familiale) et de la continuité. Cette notion de continuité se traduit, dans le système verbal de la publicité, par la façon dont le programme électoral est présenté. Au début du spot, Bush nous dit :

America's wartime economy is changing (état transitoire) to a peace-time economy. But that change has brought (aspect du passage résultatif d'un état à un autre) fear and uncertainty [...]. But with change comes (virtualité, effet de stabilisation) opportunity. And my Agenda can be (possibilité) a bridge to the future. It provides (performatif)...

Dans ce passage, l'effet transitoire indésirable (puisque la transition en question provoquera la peur et l'incertitude) s'estompe au profit d'une impression de stabilisation amenée par un glissement vers la virtualité et le performatif. L'ensemble des publicités de Bush sera caractérisé par cette préférence pour le présent virtuel et/ou performatif et la quasi-absence de la forme be + ing.

\section{Bill Clinton, renouveau et changement}

Pour Bill Clinton le problème était différent : c'était un inconnu sur le plan national. Il dut faire d'abord une campagne de quatorze mois pour gagner les élections primaires. Pendant cette période, il fut attaqué par les opposants de son propre parti (Tsongas, 
Harkin et Brown, tous candidats eux-mêmes) et par les Républicains qui lancèrent les rumeurs concernant ses liaisons extra-conjugales. La publicité proposée est l'une des premières diffusées à l'ouverture de la campagne officielle en septembre 1992.

Cette publicité tranche nettement avec celle de Bush. Elle est centrée uniquement sur le candidat qui est mis en scène dans une situation informelle : on le voit dehors, en bras de chemise, au milieu d'une foule. L'impression de dynamisme et de jeunesse domine; le mouvement de la caméra, qui passe constamment de la foule au candidat et vice-versa, souligne l'impression d'action. À ces impressions vient s'ajouter une musique rapide et "synthétique ", bâtie en une série de phrases culminantes qui souligne des idées-clés du texte et qui se termine en crescendo final sur une image de Clinton levant le bras, tel un chef d'orchestre.

11 Le message vise à distinguer Clinton non pas de son adversaire républicain comme on pouvait le supposer, mais des "anciens » Démocrates, c'est-à-dire les trois candidats ayant perdu les élections présidentielles de 1980 (Jimmy Carter), de 1984 (Walter Mondale) et de 1988 (Michael Dukakis). Le narrateur nous dit au cours du message publicitaire :

They're a new generation of Democrats, Bill Clinton and Al Gore

And they don't think the way the old Democratic Party did

They've rejected the old tax-and-spend politics

They've proposed a new plan investing in people

Ces idées sont soulignées par le slogan final :

Clinton/Gore, For people, for a change

On notera l'opposition old/new et l'utilisation du prétérit did dans la phrase «they don't think the way the old Democratic Party did ". Ces éléments font partie de l'organisation du discours chez Clinton en deux sphères: celle du MOI comprenant le candidat et l'électorat, tout ce qui est nouveau et/ou désirant le changement; celle du NON-MOI, zone d'exclusion comprenant tout ce qui est vieux y compris à l'intérieur de son propre parti. L'emploi du prétérit ici représente le seul cas du genre dans les publicités positives de Clinton; il est donc important pour lui de rejeter fermement l'image du Parti démocrate avant toute autre chose. Cette association prétérit/exclusion sera l'un des principaux aspects de la publicité négative «anti-Bush» dont nous étudierons un exemple plus loin.

Sur le plan verbal, il est également intéressant de noter que cette publicité est dominée par l'emploi de l'aspect have + en qui sert à souligner l'aspect du passage résultatif d'un état à un autre. Mais, contrairement à Bush qui ne veut pas être jugé sur les résultats (peu brillants, il faut le dire) de son premier mandat, Clinton insiste sur les notions du changement et de ses résultats qui ne sont pas négatifs, mais porteurs d'espoir.

\section{Les thèmes-miroirs : étude contrastive de deux publicités négatives}

14 La façon dont chaque candidat se perçoit et se vend aura une incidence directe sur les moyens employés pour attaquer l'adversaire dans les publicités négatives. Il convient ici de rappeler qu'il s'agit d'un spot dont le seul objectif est de détruire l'image du rival par le biais de comparaisons implicites qui renvoient aux publicités positives de l'auteur de l'attaque. Les publicités prises à titre d'exemple illustrent les deux stratégies principales employées : la dissuasion et le détournement.

Publicité anti-Clinton : la menace de l'utopie négative 
15 À l'opposé des belles images que nous proposait la publicité de George Bush analysée plus haut, cette publicité anti-Clinton est tournée en noir et blanc et montre un paysage désertique alterné avec des images accélérées d'un ciel orageux. Le hurlement du vent domine le fond sonore qui est interrompu de temps à autre par d'autres bruits : au début jusqu'à "in his state's history", on entend de la pluie et un bruit de feuilles qui volent; soudain on voit un éclair qui transperce le ciel noir, suivi du tonnerre qui sert de transition à « And yet his state remains... " puis chaque chiffre donné est ponctué par un coup de vent supplémentaire. L'ensemble de la publicité est lu par une narratrice, d'une voix monotone sans émotion. L'atmosphère menaçante du spot cherche à faire peur et à placer Clinton dans une zone "dangereuse"; il est symbolisé à la fois par l'orage dévastateur qui s'abat sur le paysage représenté et par le vautour qui guette l'horizon dans la scène finale servant d'arrière-plan au slogan America can't take that risk.

$\mathrm{Au}$ niveau sémantique nous pouvons noter rapidement deux tactiques rhétoriques de dissuasion :

17 - l'accumulation de mots chargés de sens négatif (ou évoquant chez l'électeur potentiel un sentiment de rejet) : debt/government spending/tax increase/45th Worst... (2 fois)/serious crime rate/risk.

18 - l'utilisation de l'ambiguïé sémantique dans la dernière phrase avant le slogan, « and now Bill Clinton wants to do for America what he has done for Arkansas ». Le choix de do for a son importance, car il sous-entend que Clinton pense bien faire en doublant la dette et les dépenses, en augmentant les impôts, etc. ; on nous invite donc à en conclure qu'il est un gestionnaire incompétent. De plus, dans le segment "he wants to do» on sent une anticipation du genre " he's looking forward to ", l'anticipation d'une bonne action qui aura en fait un résultat désastreux (cf. images et fond sonore). En somme, cette publicité nous menace de l'utopie négative que deviendraient les États-Unis dans l'éventualité où Clinton serait élu.

\section{Publicité anti-Bush : fiction et réalité}

19 La publicité dont il est question ici est sans doute le meilleur exemple de manipulation des temps et d'effets stylistiques au service d'un message négatif. Elle comporte plusieurs éléments chers à Clinton: un effet circulaire produit par l'emploi d'une question rhétorique avec ellipse au début et à la fin («Remember?/How're you doing?») ; des microtextes présentés à l'écran pour contrebalancer le message oral de Bush ; le détournement des propos et de l'image de l'adversaire.

20 À l'ouverture de la publicité, la question «Remember? » a un effet nostalgique et nous projette dans le passé. On s'attend à la présentation de souvenirs agréables et on peut dire ici qu'au premier degré c'est le cas, puisqu'il s'agit d'une promesse électorale pour une vie meilleure (" if you elect me President you'll be better off four years from now than you are today ", nous dit George Bush). Le choix de cette phrase permet d'amplifier l'impact de la déclaration pour trois raisons :

21 - elle est au présent et contient deux marqueurs temporels (now et today), la situant dans le «maintenant » qu'est la publicité elle-même. Elle peut prêter à confusion : on ne sait pas si l'on écoute une vieille promesse ou une nouvelle ;

22 - elle ramène Bush à un statut de candidat déchu de sa qualité présidentielle, puisqu'en 1988 il se présentait pour la première fois ; 
le cadre de la future Maison des langues, un service interuniversitaire de l'Université Stendhal-Grenoble 3 qui ouvrira ses portes officiellement en octobre 1995. Ce cours, intitulé «Le discours politique nord-américain » comprenait deux parties distinctes: le discours du multiculturalisme au Canada, et la publicité électorale comme phénomène culturel aux États-Unis. Le public visé était celui des élèves-ingénieurs de bon à de très bon niveau, recrutés sur lettre de motivation.

bjectifs pédagogiques étaient de deux ordres : didactiser une recherche théorique dans les domaines de la culture et de l'analyse du discours et proposer un enseignement de spécialité à des non-spécialistes, et ce dans la langue de la culture-cible. 
31 Mais pourquoi proposer des cours de « civilisation » à des élèves-ingénieurs, par exemple, demanderont certains? Personne n'ignore la nécessité d'apprendre aux étudiants des filières scientifiques et pluridisciplinaires la culture de la communauté professionnelle à laquelle ils seront un jour confrontés. De la même manière, il nous semble important que ces mêmes étudiants puissent acquérir ou approfondir leurs connaissances au sujet de la culture - ou plutôt des cultures - de la langue étrangère étudiée.

Dans un cours de ce type, les enjeux pédagogiques sont de taille. Il faut trouver un équilibre entre les cours centrés sur l'apport d'informations de base («background information ») et les cours privilégiant l'interactivité. Nous avons décidé de fournir une partie des éléments historiques et géo-politiques sous forme de fascicules composés de documents authentiques. Dans le cas de la publicité électorale aux États-Unis, les éléments historiques donnés étaient les suivants : the Declaration of Independence, the U.S. Constitution (document complet et une synthèse des points saillants), cartes géographiques illustrant le développement et l'expansion du territoire des premières colonies à nos jours et articles journalistiques : remise en cause du "melting pot », articles d'opinion (editorials) sur la campagne présidentielle, tableau récapitulatif des résultats de l'élection.

33 Les étudiants étaient invités à lire ces documents avant le début du module ; lors de la première séance, nous avons travaillé à partir de trois documents sonores - the Pledge of Allegiance, the Star-spangled banner (hymne national) et « 0 , beautiful » (autre chanson très connue aux États-Unis). L'ensemble des documents a servi de base de discussion sur les valeurs de la société américaine; il est intéressant de noter que la plupart des participants au cours avaient déjà séjourné aux États-Unis et souhaitaient apporter leurs impressions afin de se faire une opinion plus «documentée ». Par ailleurs, l'étude des images présentes dans les deux chansons a servi de point de départ à l'analyse des images des publicités politiques.

Pour la phase d'analyse des publicités, les étudiants disposaient d'un autre fascicule contenant la transcription d'une vingtaine de spots. Si les étudiants n'avaient pas de mal à interpréter le symbolisme iconographique (compte tenu du travail préparatoire fait en amont), il n'en était pas de même pour l'analyse du discours proprement dit; il a fallu leur apporter des éléments techniques et une terminologie appropriée pour ce travail. Quelle ne fut pas leur surprise de découvrir que l'on peut analyser «scientifiquement " une langue!

En fin de parcours (dix séances de $1 \mathrm{~h} 30$ chacune, dont la moitié consacrée aux publicités électorales), les étudiants ont produit un rapport de synthèse de cinq pages sur un sujet de leur choix, qu'ils ont présenté oralement en quinze minutes devant l'enseignant et les étudiants du groupe.

Si le bilan de cette expérimentation est globalement positif, il reste encore des problèmes à régler: il faut que cette option, lorsqu'elle est choisie par l'étudiant, puisse être reconnue et validée par l'établissement d'origine, même si l'étudiant souhaite la suivre en plus de sa formation normale en langues. Par ailleurs, il faut continuer à réfléchir sur la manière d'apporter le plus d'informations possibles à l'étudiant sans compromettre l'interactivité. Enfin, il faut privilégier le développement de la recherche appliquée dans le domaine culturel, ce qui permettra l'enseignement d'autres cours de ce type. 


\section{BIBLIOGRAPHIE}

Donovan, R. \& R. Scherer, R. 1992. Unsilent Revolution: Television news and American public life. Cambridge : Cambridge University Press.

Kern, M. 1989. 30-Second Politics: Political advertising in the eighties. New York : Praeger Publishers.

Le Monde diplomatique. 1989. La communication victime des marchands : affairisme, information et culture de masse. Paris : La Découverte/Le Monde.

Ramonet, I. 1989. « Télévision et politique ». In Le Monde diplomatique, La communication victime des marchands : affairisme, information et culture de masse. Paris : La Découverte/Le Monde.

\section{ANNEXES}

\section{Transcription des publicités étudiées}

\section{Publicités positives}

1.1. (Bush est dans son bureau à la Maison-Blanche. Derrière lui; on voit un drapeau américain ; on aperçoit le jardin à travers une fenêtre)

Today, for the first time in half a century, America is not at war. And America's wartime economy is changing to a peace-time economy. (image du défilé de la victoire après l'opération Desert Storm ) But that change has brought fear and uncertainty: the fear of losing a job, of losing a home; the uncertainty of tougher economic competition from around the world. But with change comes opportunity. And my Agenda for American Renewal (Bush assis à son bureau en train d'écrire, sa brochure sur un côté) can be the bridge to the future. It provides a choice of quality schools (image d'une salle de classe, les enfants tournent le dos à la caméra pour être face au drapeau américain) so our children have the knowledge to compete. It provides job training (2 femmes assises devant un ordinateur, dont une est infirmière) so workers have the new skills to compete. It provides health care for all (image d'une femme enceinte) and controls spiralling costs so our people can afford to compete (même femme avec le bébé dans les bras). And my agenda strengthens small businesses ('barber shop' dont le rideau s'ouvre) so we have the new jobs to compete (jeune homme noir distingué au téléphone). Today we stand at the threshold of a new era (ouvrier dans une usine sidérugique). We changed the world around us. Together we must do the same at home. (Bush et sa femme habillés en vêtements de sport, en train de se promener dans la nature entourés de leurs petitsenfants.)

\section{BUSH/QUAYLE '92}

1.2. (Clinton et Gore sont au milieu d'une foule, en train de serrer la main à des admirateurs ; images qui bougent, de style vidéo amateur) They are a new generation of Democrats, Bill Clinton and Al Gore, and they don't think the way the old Democratic Party did. They've called for an end to welfare as we know it, so welfare can be a second chance, not a way of life. 


\section{Welfare can be a second chance}

\section{Not a way of life}

They've sent a strong signal to criminals by supporting the death penalty

Support the death penalty

And they've rejected the old tax-and-spend politics. Clinton has balanced twelve budgets Bill clinton has balanced twelve budgets

And they've proposed a new plan investing in people, detailing 140 billion dollars in spending cuts they'd make right now.

Cut $\$ 140$ Billion in wasteful spending

Clinton/Gore: for people, for a change.

Clinton/Gore: For people, for a change

(dernière image avant le slogan : 2 Noirs sur un tracteur au bord de la route tenant un panneau « Clinton/Gore ' 92 » et brandissant un drapeau américain)

\section{Publicités négatives}

2.1. (Voix d'une narratrice; publicité en noir et blanc : la scène consiste en des images de ciel orageux, de paysages lunaires, arides. On entend le tonnerre et le vent. Les textes en gras apparaissent à l'écran.)

In his twelve years as governor of Arkansas, Bill Clinton has

Doubled his state's debt

Doubled government spending

signed the largest tax increase in his state's history

The largest tax increase in state's history

Yet his state remains the

45th Worst in Which to Work*

*Source: The Corporation for Enterprise Development

(the) 45th Worst for Children*

*Source: The Center for the Study of Social Policy

(it has the) Worst Environment Policy*

*Source: The Institute for Southern Studies

and the FBI says Arkansas had America's biggest increase in the rate of serious crime

Biggest Increase in Serious Crime Rate*

*Source: The FBI

And now Bill Clinton wants to do for America what he's done for Arkansas.

America can't take that risk.

\section{2. (écran télé sans image) Remember?}

(image vidéo de Bush, publicité politique de la campagne 1988)

Bush: And if you elect me President, you will be better off four years from now than you are today.

(Narrateur + texte à l'écran)

Average family income down $\$ 1,600$

in two years

Commerce Department

Bureau of Census 9/3/92

(même image de Bush d'un peu plus près) 
Bush: You will be better off four years from now than you are today.

(Narrateur + écran)

Family healthcare costs up $\$ 1,800$

in four years

Health Insurance Association of America 1988

KPMG PEAT MARWICK 1992

Bush (zoom sur visage): You'll be better off four years...

(Narrateur + écran)

The second biggest tax increase in history

Congressional Budget Office Study 1/30/91

New York Times 8/7/92

Bush (zoom sur images, qui sautent) And if you elect me President, you'll be better off four years from now than you are today.

(Retour à l'écran sans image du début ; texte et voix de narrateur)

Well, it's four years later.

How're you doing?

\section{NOTES}

1. Unique Selling Proposition en anglais

2. Voir annexe pour les textes des quatre publicités ainsi que quelques indications scéniques.

3. Dans son ouvrage, Montague Kern parle de "swelling music which tells a story through sound, a sound that evokes the memory of myth and stories in childhood [...]. The music reinforces the visuals, beginning tentatively, rising, then moving to a climax, leaving the viewer with the feeling something has been settled in a happy ending » $(1989: 82)$.

\section{RÉSUMÉS}

Quel est le rôle de la publicité électorale aux États-Unis et quel est son impact sur le résultat du scrutin? Cette présentation tentera de répondre à cette question en analysant, dans un premier temps, les principaux éléments linguistiques et culturels de ce moyen de communication bien particulier à travers quatre exemples enregistrés à la télévision américaine pendant la campagne présidentielle de 1992. Dans un deuxième temps sera analysée la didactisation de cette recherche pour l'enseignement de la moitié d'un module optionnel intitulé «Langue et civilisation contemporaines : le discours politique nord-américain ». La particularité de ce cours consiste à enseigner une matière dite de spécialité aux non-spécialistes, dans notre cas des élèvesingénieurs de divers horizons, en privilégiant l'interactivité, le travail de groupe et l'expression orale formelle et informelle.

What role does campaign advertising play in the race for the White House? This paper first proposes to answer the question by analysing the predominant linguistic and cultural features of four political ads broadcast during the 1992 presidential campaign that opposed George Bush and 
Bill Clinton. Secondly, the teaching methods developed to enable the use of this material in an experimental course for non-specialists are examined.

INDEX

Keywords : advertising, language of persuasion, cultural symbolism, interactive teaching method, presidential campaign

Mots-clés : campagne présidentielle, didactisation, langue de la persuasion, publicité, symbole culturel

\section{AUTEUR}

DONNA ANDRÉOLLE

Donna Andreolle enseigne à Université Stendhal Grenoble 3. donna.andreolle@univ-lehavre.fr 